\title{
Sensor design and implementation for a downhole NMR fluid analysis laboratory
}

\author{
Wu Baosong, Xiao Lizhi*, Li Xin, Yu Huijun and An Tianlin \\ State Key Laboratory of Petroleum Resource and Prospecting, China University of Petroleum, Beijing 102249, China \\ (C) China University of Petroleum (Beijing) and Springer-Verlag Berlin Heidelberg 2012
}

\begin{abstract}
This paper tries to build a multi-functional downhole nuclear magnetic resonance (NMR) fluid analysis laboratory that can evaluate fluid information in real time at reservoir conditions at a depth of several thousand meters. The aim is to monitor the pollution of the formation fluids and quantitatively evaluate NMR characteristics of the fluids. It focuses on the design of the structure and parameters of a sensor with zero stray fields. Two separate coils are designed to measure NMR characteristics of flowing or static fluids. A method is proposed to use the Bloch equation, to guide the optimization of the NMR sensor. Finally, the measured results confirm that the design is reasonable. There is a homogeneous static field (perpendicular to the axial direction) in the center of the sensor, and there are no stray external fields. The novel design of pre-polarization magnet improves the signal to noise ratio, while shortening the sensor length.
\end{abstract}

Key words: NMR, fluid analysis laboratory, magnet, Bloch equation

\section{Introduction}

Oil and gas is a fluid resource often at depths of several thousand meters. A multi-functional fluid laboratory that can be used to analyze the reservoir fluids in situ real-time is a consistent goal of petroleum scientists. A downhole NMR fluid analysis laboratory can achieve these logging needs in the well bore. It can determine the composition and properties of fluids sampled by the Reservoir Description Tool (RDT).

Currently, the methods that could precisely provide many quantitative parameters about formation fluid are the optical analysis method and NMR method. The former is based on the optical principles of reflection and transmission to identify gas and fluid properties in flow lines, and to determine the fluid composition (Dong et al, 2007). However it does less well when the filtrate is oil based or when the formation fluid is a mixture of oil and water and mixed-phase fluids are very common in oil production. NMR relies on the response of atomic nuclei to magnetic fields to obtain fluid information at reservoir temperature and pressure conditions (Akkurt et al, 2004; Minh et al, 2008). The technique is not affected by filtrate or reservoir and can not only monitor contamination from filtrate, but also can acquire a variety of NMR parameters of the formation fluids (Prammer et al, 2001; Seifert et al, 2007).

NMR technology is inherently noninvasive and noncontacting and has been widely applied as a testing method in many fields. There are three main application areas of NMR.

\footnotetext{
* Corresponding author. email: xiaolizhi@cup.edu.cn Received April 2, 2011
}

These are relaxometry, imaging and spectroscopy (Blümich et al, 2011). NMR measurements have become an important part of oilfield well logging to identify and quantify oil and gas reservoirs. Because of the harsh environment in the borehole, we obtain formation information by means of NMR relaxometry only, which concerns the measurement of timedomain signals that bear information about relaxation and diffusion. There are three types of NMR logging tool: wireline NMR, logging-while-drilling (LWD) NMR and NMR fluid analysis laboratory (Coates et al, 1999). In practical applications, the NMR fluid analysis module, combined with the RDT, is lowered to the required well section for fixedpoint measurements. It yields information for determining fluid NMR properties at reservoir conditions, such as spinlattice relaxation time $\left(T_{1}\right)$ or spin-spin relaxation time $\left(T_{2}\right)$.

NMR technology measuring relaxation and diffusion of fluids is very mature, but it is difficult to move the "mature" laboratory five kilometers down a borehole. This is because a downhole NMR instrument has to withstand hostile conditions such as high temperature $\left(175^{\circ}\right)$, high pressure $(20,000 \mathrm{psi})$, and high mechanical shock. In addition, the instrument must be designed to fit inside a small hole (typically 6-12 inches in diameter) (Luong et al, 2001). So development of a downhole NMR fluid analysis laboratory has always been technically difficult for the petroleum industry. There are some patents and articles that discuss different designs of the downhole NMR fluid analysis laboratory (Blades and Prammer, 2000; Edwards et al, 2000; Prammer et al, 2002; Joo, 2009). This paper is based on the sensor configuration of Prammer et al (2002), and focuses on improving the design of the sensor and associated studies. 


\section{Theory}

The schematic diagram of NMR fluid analysis laboratory is shown in Fig. 1. According to its working process, we design a workflow for the NMR fluid analysis laboratory, as shown in Fig. 2. The sensor module in the RDT pulls the formation fluids into the flow line. In the initial stage, the flow fluids run through the NMR analysis module and then into the borehole. There is a need for a method that monitors contamination of the original formation fluids in this process. We measure the $T_{1}$ parameter to evaluate this fluid property. When the formation fluid properties are stable (contamination has been reduced to a minimum), the transverse relaxation time $T_{2}$ and the diffusion coefficient $D$ of fluids in stagnate are measured. Then quantitative analysis is undertaken, which could be used to quantitatively evaluate magnetic resonance characteristics of the fluids; the fluids in the flow line are sampled or expelled to the borehole at the end of the measurement.

NMR refers to the response of atomic nuclei to magnetic fields. In our applications, permanent magnets and an antenna coil provide the necessary static and time-varying RF magnetic fields. Fig. 3 depicts the schematic circuit diagram of the NMR fluid analysis laboratory. As shown in Fig. 3, fluids run through the tube, $B_{0}$ field is perpendicular to the axial direction, but $B_{1}$ field is along it. Digital signal processor controls the signal generator which generates RF pulse sequence sent to RF transmitter coil, while processes the acquired echoes. In transmit mode, signal runs through the power amplifier to form high voltage RF pulses. The pulse will turn on the diode, so that the two coils (Coil 1, Coil 2) are used as RF coil forming $B_{1}$ field which is parallel to

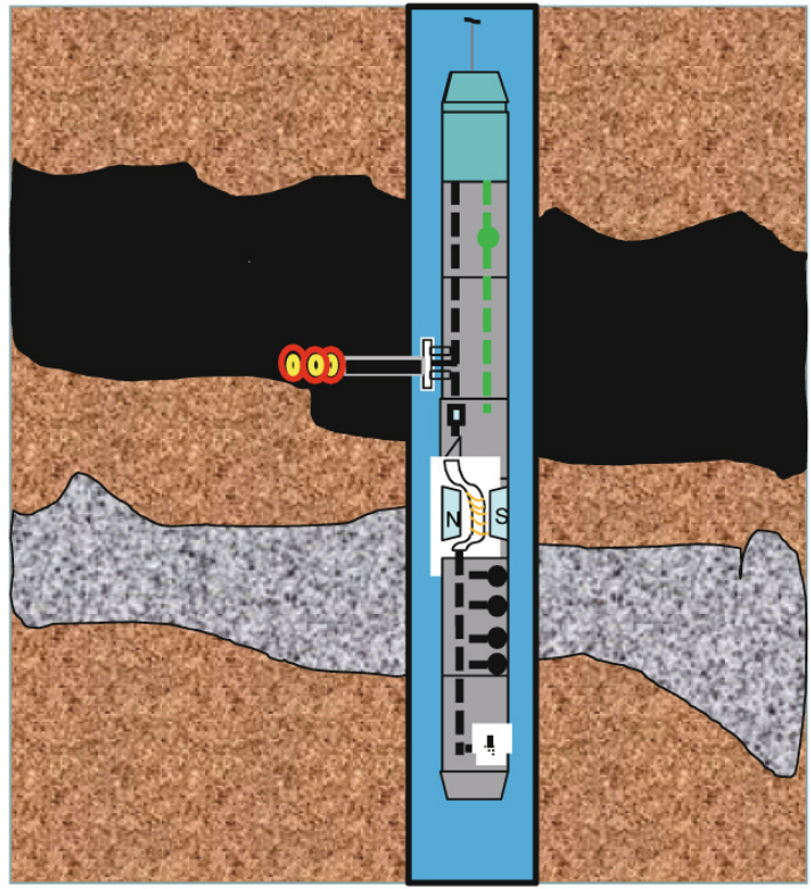

Fig. 1 The schematic diagram of NMR fluid analysis laboratory module combined with the RDT. The tool is lowered to the required well section for fixed-point measurements. The formation fluids are pulled into the flow line through the sink probe, and then enter NMR fluid analysis laboratory the axial direction in the tube. In receive mode, echo signal is too small to turn on diodes, that is, Coil 1 is in isolation, only the induce signal in Coil 2 passes through isolation circuit. The signal is amplified, digitized and then fed into the digital signal processor for further processing.

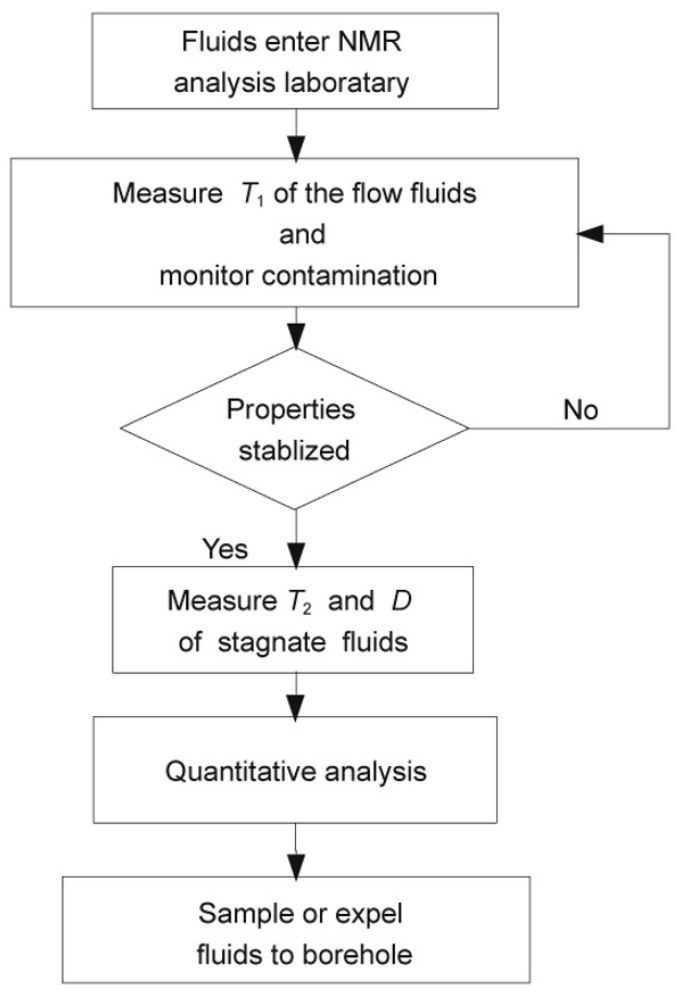

Fig. 2 The flow chart of the NMR fluid analysis laboratory

\section{Sensor structure and parameters design}

The sensor structure shown in Fig. 4 is a key part for downhole NMR testing of formation fluids. It comprises: magnets in the polarization section (part A and part B) for fast polarizing hydrogen nuclei, magnets in resonance section (part C) for resonance measurement, two separate coils for generating the oscillating magnetic field and receiving echoes, a vessel for providing a passage for fluid, a steel tube with high magnetic permeability. The static magnetic field in the vessel is substantially uniform with a defined magnetic field direction. The formation fluid entering the sensor is initially subjected to a strong magnetic field to achieve a rapid polarization, and then enter the resonance section to measure $T_{1}$ while the fluid is flowing.

The static magnetic field $B_{0}$ is perpendicular to the central axis, and RF field $B_{1}$ is parallel to the axis. Coil 1 is used for transmitting only, while Coil 2 both transmits and receives. We can obtain $T_{1}$ information with saturation recovery (SR) sequences which will be described in Fig. 11 while the flow rate of fluids is within a certain range. To perform $T_{2}$ and selfdiffusion coefficient $D$ measurement, the fluid in the sensor is held static within the NMR chamber.

There are many methods for designing the magnets for a uniform field in the center (Prammer et al, 2002; Joo, 2009; Miller et al, 2002). But for the fluid analysis laboratory, the magnet needs to meet two conditions: firstly, the space in the 
well borehole is small, so the magnet must be small enough and easy to install; secondly the uniform region should be big and the magnetic field variations are as small as possible, preferably not exceeding $0.1 \%$, and there should be no external stray fields. Fig. 5 shows the cross-sectional view of the resonance region in the sensor (the cross-sectional structure of pre-polarization magnet is the same).

The entire magnet consists of three parts. Each part is built by stacking several ring magnet segments along the axial direction as shown in Fig. 4. One of the important objectives in designing an NMR sensor is to produce a strong and homogeneous field $B_{0}$ in the center region (see Fig. 5). The magnetic flux density field $B$ generated by the permanent magnet satisfies the equations:

$$
\left\{\begin{array}{l}
\nabla \times\left(\frac{1}{\mu} B\right)=\nabla \times M \\
\nabla \cdot B=0
\end{array}\right.
$$

where $M$ is the net magnetic dipole moment per unit volume, and $\mu$ is the permeability.
In Fig. 5, the arrows indicate the direction of magnetic polarity, pointing from $\mathrm{N}$ to $\mathrm{S}$. Through the cross section, we know that the key section of the sensor includes a vessel that is made of Poly-Ether-Ether-Ketone (PEEK), a slotted copper tube, samarium-cobalt permanent magnets, a nonslotted copper tube, a high magnetically permeable steel tube and glass shell (to prevent corrosion and wear of the steel, not shown). The slotted copper tube, located between the vessel and solenoid coil, shields any electric field and non-axial RF field generated by the coils. The magnet elements are attached to the non-slotted copper, which lets the static magnetic field pass and shields any dynamic electromagnetic field.

The permanent magnet produces a uniform static magnetic field in the vessel. The magnet is encased in a magnetically permeable steel tube that acts as magnetic shield and confines the field to the inside of sensor and provides a closed magnetic circuit to enhance the field in the destination area. The reluctance of the glass tube or air is much greater than the reluctance of ferromagnetic materials. The magnetic lines of force will run along the steel tube wall and then return back to the sensor, so the external field strength is weak.

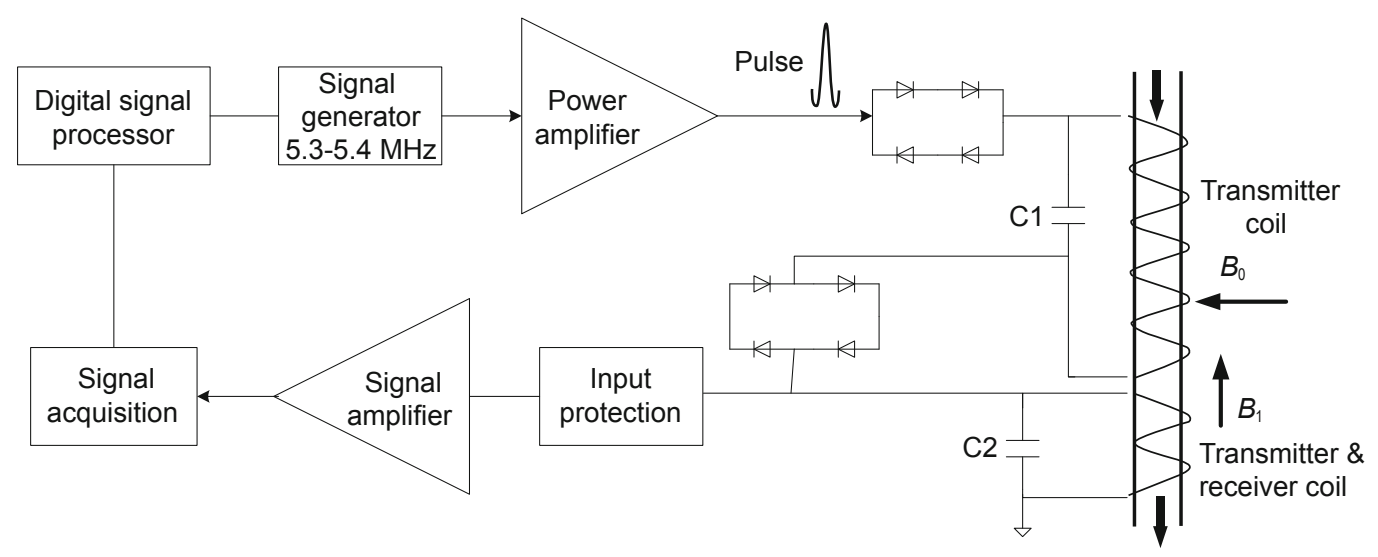

Fig. 3 The schematic circuit diagram of the NMR fluid analysis laboratory

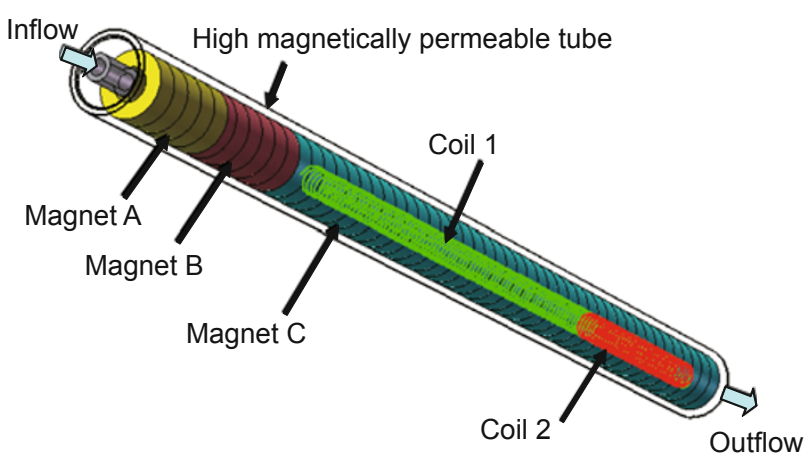

Fig. 4 The sensor structure

\subsection{The thickness of the copper}

In above paragraph, we described the functions of the slotted and non-slotted copper tubes. The magneto-acoustic ringing in copper tube should be as small as possible. There are two ways to reduce the ringing effects in pulsed NMR: avoiding gross motion of the conductor and suppressing ultrasonic waves. The gross motion is relatively easy to deal with. We can restrain the coil or the shield with RTV silicone adhesive. The problem is most serious when the frequency of the ultrasonic wave is coincident with a shear or compressional mode of the structure. Standing waves will occur whenever the thickness is equal to an integer number of half of wavelengths. The thickness modes of a metal plate occur at frequencies given by $f=m \times s / 2 d$, where $m$ is an integer, $s$ is the shear or compressional speed of the material, and $d$ is the thickness of the plate (Buess and Petersen, 1978; Fukushima and Roeder, 1979). We use copper as the shield. The compressional speed in rolled copper is $5,010 \mathrm{~m} / \mathrm{s}$ and the shear speed is $2,270 \mathrm{~m} / \mathrm{s}$. To ensure that the lowest thickness mode is above $5.36 \mathrm{MHz}$, a copper plate must be no thicker than $0.2 \mathrm{~mm}$ ( $>3$ times the skin depth of copper at $5.36 \mathrm{MHz}$ ). Meanwhile, the shield should be connected to the ground of the sensor. 

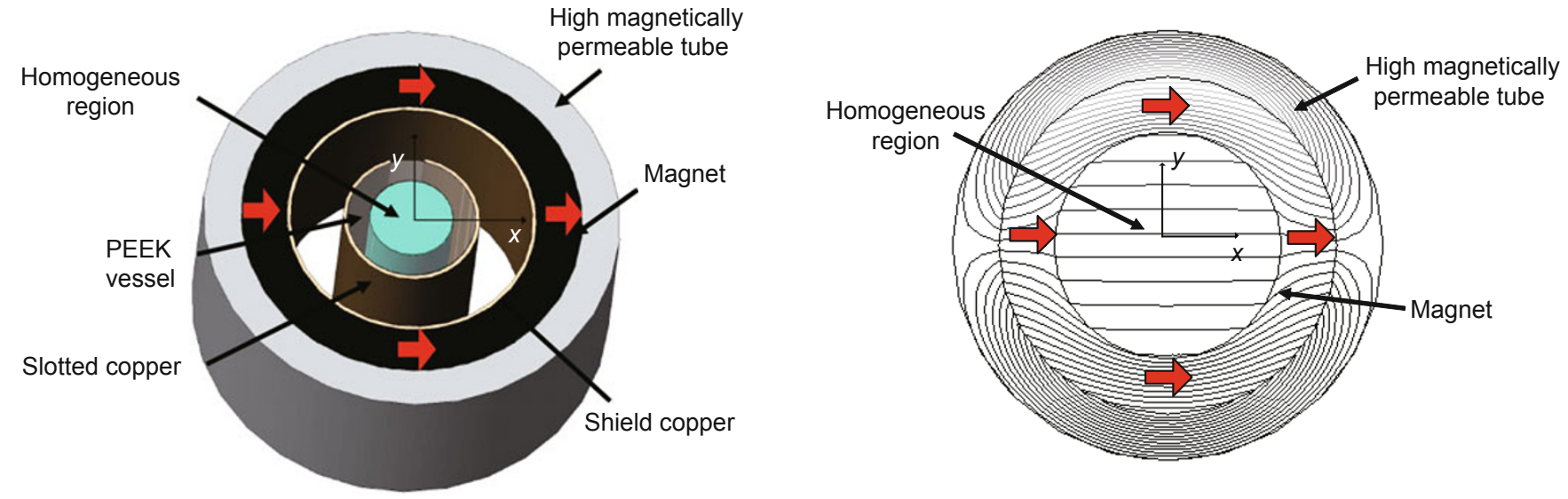

Fig. 5 The cross section of the resonance region

\subsection{The material and thickness of the high magnetically permeable tube}

The logging tools are constrained by the size of borehole, so we must consider the thickness of a steel tube with high magnetically permeability. In Fig. 6, it can be seen that the static field $B_{0}$ in the center of the sensor changes with tube thickness and magnetic permeability of the selected material. The results are obtained by means of finite element method (FEM) simulation. The red line represents that the tube thickness is $12 \mathrm{~mm}$, the green line is $10 \mathrm{~mm}$, and the blue line is $5 \mathrm{~mm}$. Compared with the thickness of the tube, the permeability of material has greater impact on strength of $B_{0}$ field. When the magnetic permeability of the material is greater than 1,000, the strength of the $B_{0}$ field is more stable. We use technically pure iron as the material of the tube. This material is a good choice because it can have a relative permeability of several thousands and will not saturate below 2 Tesla, well above the surrounding field. Even if the permeability changes a little at high temperatures, it has negligible effects on the field. We should also consider the high pressure on designing the thickness in the downhole. Besides adjusting thickness of the magnet, we can change the gap between steel tube and permanent magnet to get the right field strength for optimal design (Miller et al, 2002).

The high magnetically permeable tube is $10 \mathrm{~mm}$ thick. The cross-section structure in Fig. 5 (left) is simulated by FEM software. In Fig. 7, the curves show the magnetic field gradient in the cross section of the resonance region. The red line indicates the gradient from $-0.31 \mathrm{~cm}$ to $0.31 \mathrm{~cm}$ along $x$-axis, and the blue line represents the gradient from -0.31 $\mathrm{cm}$ to $0.31 \mathrm{~cm}$ along $y$-axis. It can be seen that there is a larger uniform field region in the middle, and the maximum gradient is less than $0.001 \mathrm{Gauss} / \mathrm{cm}$ within a radius of about $11 \mathrm{~mm}$. The static field is homogeneous enough to meet our requirements.

\subsection{Determine the lengths of magnet}

Pre-polarization magnet

The magnetic field varies along the axial direction. Magnets are comprised of three parts: pre-polarization magnet A (magnetic field strength of about 2,700 Gauss in the center region), pre-polarization magnet $\mathrm{B}$ (about 1,010 Gauss) and

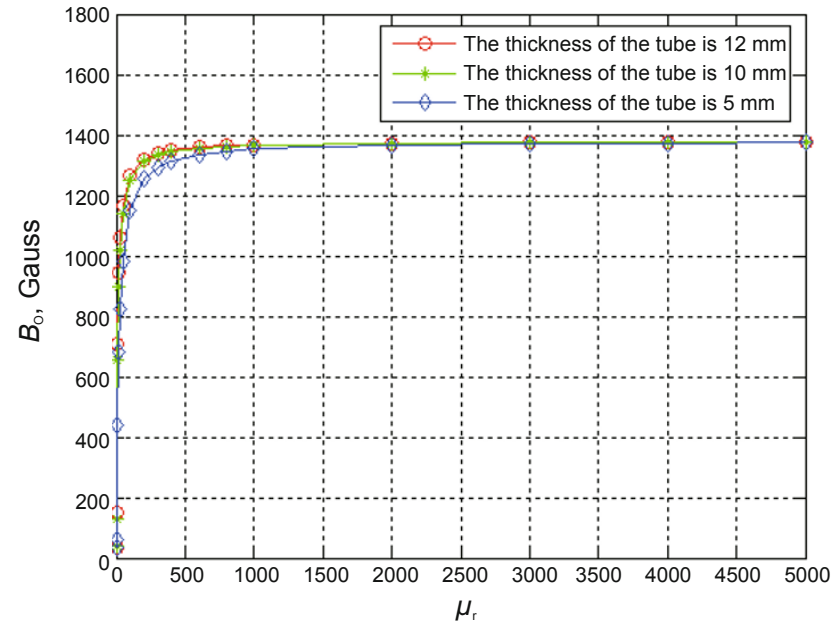

Fig. 6 The static field $B_{0}$ changes with the tube thickness and magnetic permeability of the selected material

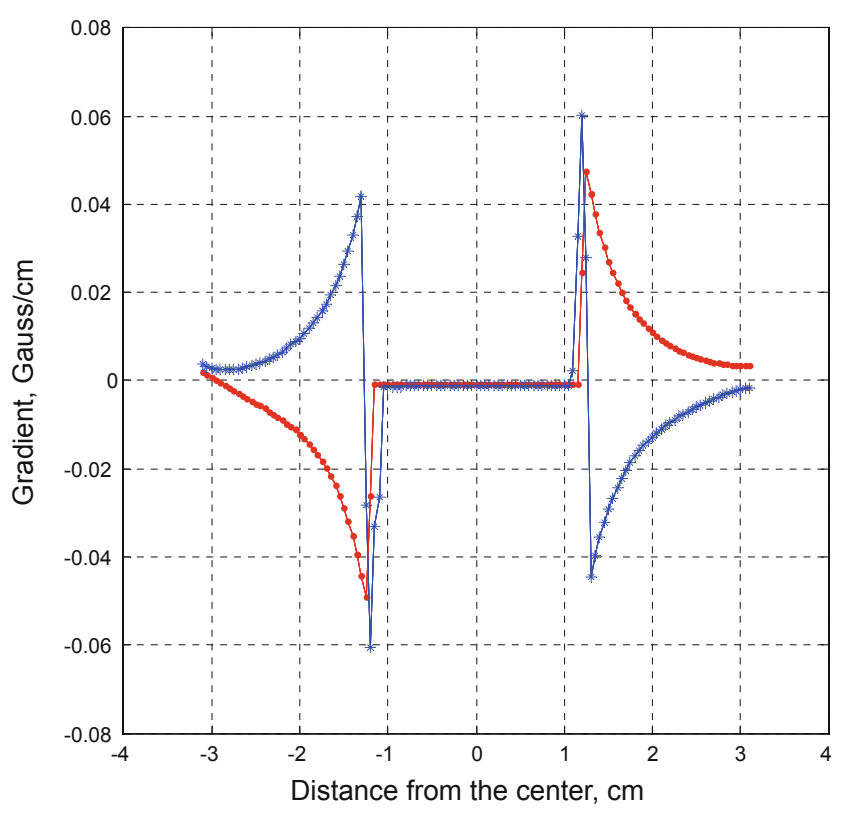

Fig. 7 Gradient changes with location in the resonance region

magnet $C$ (about 1,260 Gauss). The pre-polarization magnet provides a "pre-polarization zone". Passing through A and B 
sections, the fluid enters the measure section $\mathrm{C}$, and then we can get information about $T_{1}$ in the course of the motion.

Here we describe how the magnetization of the fluids changes as they pass through the magnet. If the velocity of fluids is $v$ when they go into part A, the length of magnet $\mathrm{A}$ is $L_{\mathrm{A}}$. The magnetization is $M\left(t_{1}\right)$ after being polarized by part A (where $t_{1}=L_{\mathrm{A}} / v$ ). During movement of the fluid into part B the magnetization decays. The length of magnet B is $L_{\mathrm{B}}$. When the fluid moves into part $\mathrm{C}$, its magnetization becomes $M\left(t_{2}\right)$ (where $t_{2}=\left(L_{\mathrm{A}}+L_{\mathrm{B}}\right) / v$ ). It can be seen clearly that polarization path is from the origin to point a, then ends at point $b$ (see Fig. 8). The magnetization of the fluids is $1.26 M_{0}$ when they flow into part $\mathrm{C}$. This will be the optimal design (suppose the final magnetization of the protons in the magnetic field 1,000 Gauss is $M_{0}$, the final magnetization in the 1,260 Gauss would be $1.26 M_{0}$ ).

In designing the magnet, we require that the magnetization of fluids is close to $1.26 M_{0}$ when they enter the measurement zone. A flow rate of $5 \mathrm{~cm} / \mathrm{s}$, ensures that the magnet length of both $\mathrm{A}$ and $\mathrm{B}$ is minimized. That is to say to minimize the sum of $L_{\mathrm{A}}$ and $L_{\mathrm{B}}$. Based on Bloch's equation (Eq. (2)), we calculate the appropriate lengths of magnet.

$$
\left\{\begin{array}{l}
\frac{\partial M_{Z}(r, t)}{\partial t}=\frac{1}{T_{1}}\left(x_{0}\left|B_{0}(r, t)\right|-M_{Z}(r, t)\right) \\
M_{Z}(., 0)=M_{i}
\end{array}\right.
$$

In Eq. (2), $M_{Z}(r, t)$ (the subscript $z$ stands for "longitudinal" component of the magnetization in a rotating frame parallel to $B_{0}$ ) is the net magnetization which is instantaneous and depends on location and time. $x_{0}$ is the susceptibility that depends only on the fluids temperature. $M_{i}$ is a known magnetization at the initial time (that is, $t=0$ ). The polarization curves are based on Eq. (2).

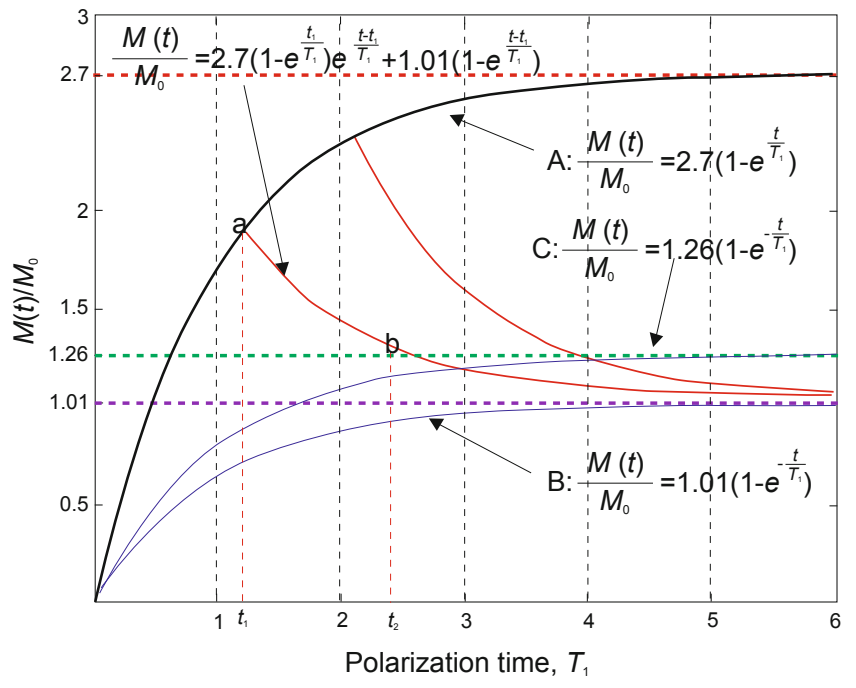

Fig. $8 T_{1}$-polarization curves indicate the degree of magnetization, as a function of the time that a proton population is exposed to an external magnetic field

Different fluids, such as water, oil, gas, have very different $T_{1}$ relaxation times. The longitudinal relaxation times of fluids are generally 2-3 seconds. Firstly, when the magnetization of fluids (relaxation time $T_{1}=3 \mathrm{~s}$, velocity $v=5 \mathrm{~cm} / \mathrm{s}$ ) is 1.3 $M_{0}$ at the entrance of magnet $\mathrm{C}$, we get a series of length combinations of the magnet A and B (see Table 1). Then let fluids with different $T_{1}$ relaxation times (range of 2-3 s) pass through combinations in Table 1 , respectively. We select the appropriate magnet length.

Each curve in Fig. 9 shows that when fluids with the same $T_{1}$ flow through the combination of different magnet lengths in Table 1, they get the net magnetization at the entrance to the measurement section. It can be seen that the focal point of curves is in the vicinity of point $(15,15.38,1.26)$. We select the 8 th combination $\left(L_{\mathrm{A}}=15 \mathrm{~cm}, L_{\mathrm{B}}=15.38 \mathrm{~cm}\right)$ in Table 1. Fluids with different relaxation times $T_{1}$ will get

Table 1 Length combinations of the magnet A and B

\begin{tabular}{|c|c|c|c|c|c|c|}
\hline & $t_{1}=\frac{L_{\mathrm{A}}}{v}, \mathrm{~s}$ & $L_{\mathrm{A},} \mathrm{cm}$ & Point a, $M(t) / M_{0}$ & $t_{2}=\frac{L_{\mathrm{A}}+L_{\mathrm{B}}}{v}, \mathrm{~s}$ & $L_{\mathrm{B},} \mathrm{cm}$ & $L_{\mathrm{A}}+L_{\mathrm{B},}, \mathrm{cm}$ \\
\hline 1 & 1.887 & 9.435 & 1.2606 & 1.887 & 0 & 9.435 \\
\hline 2 & 2.1 & 10.5 & 1.359 & 3.113 & 5.07 & 15.57 \\
\hline 3 & 2.5 & 13.5 & 1.527 & 4.677 & 9.89 & 23.39 \\
\hline 4 & 2.8 & 14 & 1.638 & 5.565 & 13.83 & 27.83 \\
\hline 5 & 2.9 & 14.5 & 1.673 & 5.825 & 14.63 & 29.13 \\
\hline 6 & 2.95 & 14.75 & 1.69 & 5.952 & 15.01 & 29.76 \\
\hline 7 & 2.98 & 14.9 & 1.7 & 6.026 & 15.23 & 30.13 \\
\hline 8 & 3 & 15 & 1.707 & 6.075 & 15.38 & 30.38 \\
\hline 9 & 3.04 & 15.2 & 1.72 & 6.172 & 15.66 & 30.86 \\
\hline 10 & 3.1 & 15.5 & 1.739 & 6.312 & 16.06 & 31.56 \\
\hline 11 & 3.15 & 15.75 & 1.755 & 6.427 & 16.39 & 32.14 \\
\hline 12 & 3.2 & 16 & 1.771 & 6.539 & 16.7 & 32.7 \\
\hline 13 & 4 & 20 & 1.988 & 8.093 & 20.47 & 40.47 \\
\hline
\end{tabular}




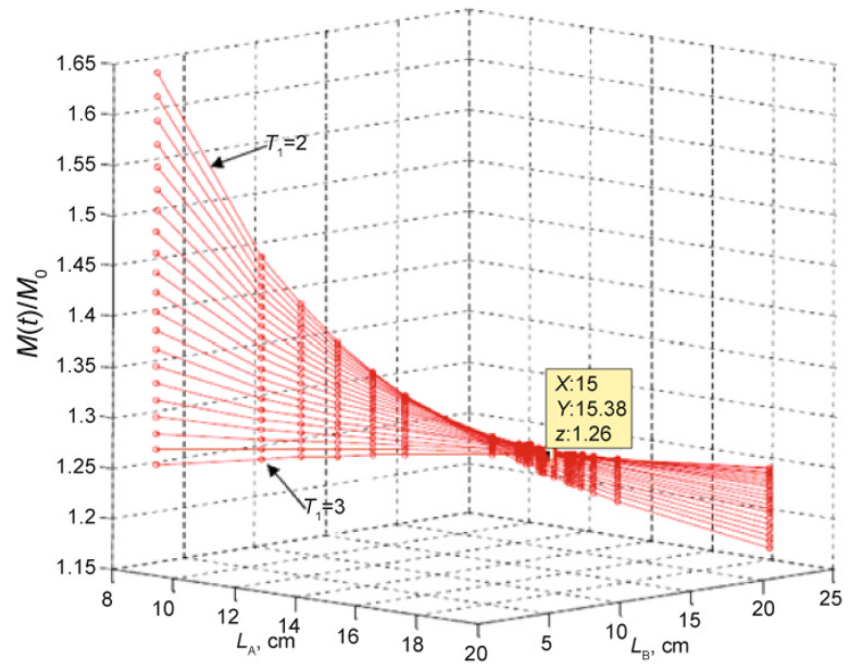

Fig. 9 The curves represent that fluids flowing through the combinations of different magnet lengths get the net magnetization. Each circle represents a combination. The $T_{1}$ relaxation time changes from 2 to 3 seconds

the magnetization of $1.26 M_{0}$ at the entrance. In practice the length of the magnet $A$ is $15 \mathrm{~cm}$, and $B$ is $15.4 \mathrm{~cm}$.

Different fluids flow through the pre-polarization magnet into the resonance region. A magnetization curve is illustrated in Fig. 10. The final magnetization in the resonance region is $1.26 M_{0}$. It can be seen that the magnetization reaches $98 \%$ of its final value as fluids pass through the design length. It indicates that the design is reasonable.

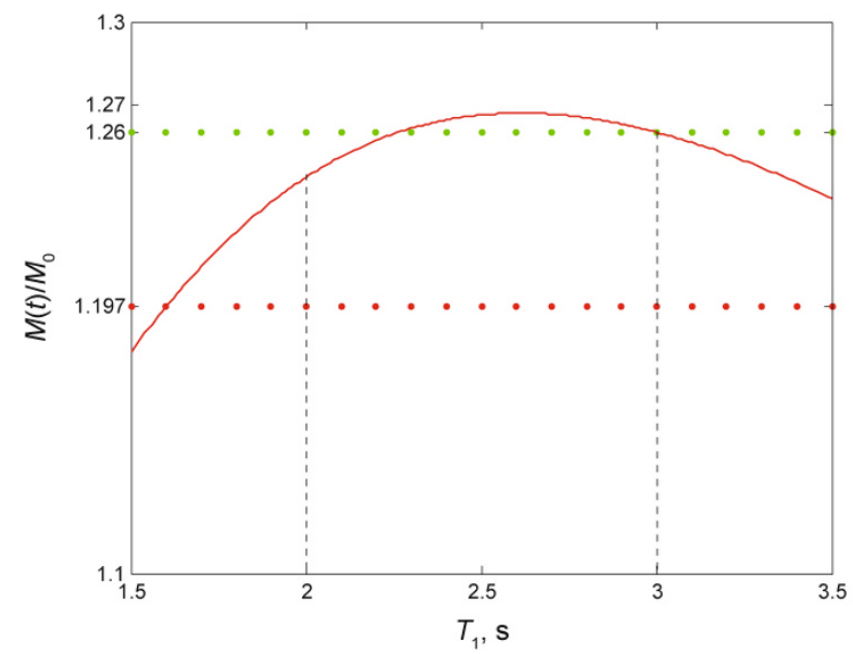

Fig. 10 The magnetization is illustrated as different fluids enter resonance region

Resonance region magnet

Fluid is flowing during the process of measuring $T_{1}$. So it is necessary that the required length of coils is longer than that as fluid in stagnation. This can make sure that fluids in the reception area are on resonance. The NMR fluid analyzer uses SR sequences to measure $T_{1}$. An initial saturation pulse followed by a wait time $t_{\mathrm{SR}}\left(t_{\mathrm{SR}}=1,2,4,8,16, \ldots, 16,384\right.$ $\mathrm{ms}$ ), varying from 1 to $16,384 \mathrm{~ms}$, results in a total cycle time of approximately 33 seconds. Fig. 11 shows the SR pulse sequence. The signal amplitude of the oil sample changes with wait time $t_{\mathrm{SR}}$ (see Fig. 12).

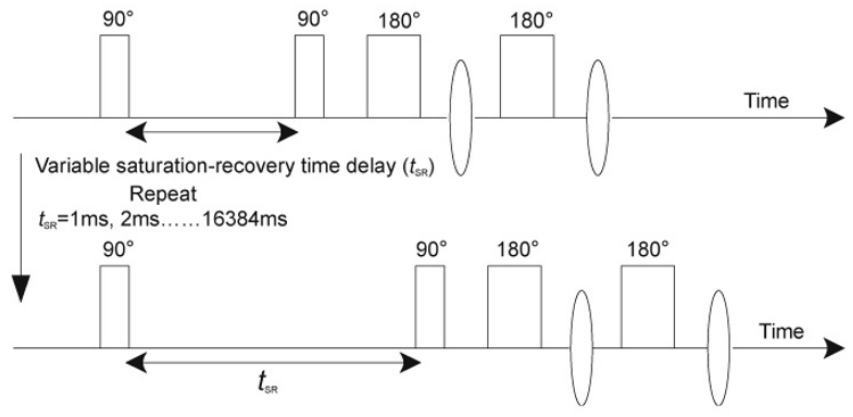

Fig. 11 The saturation recovery sequences for $T_{1}$ measurement

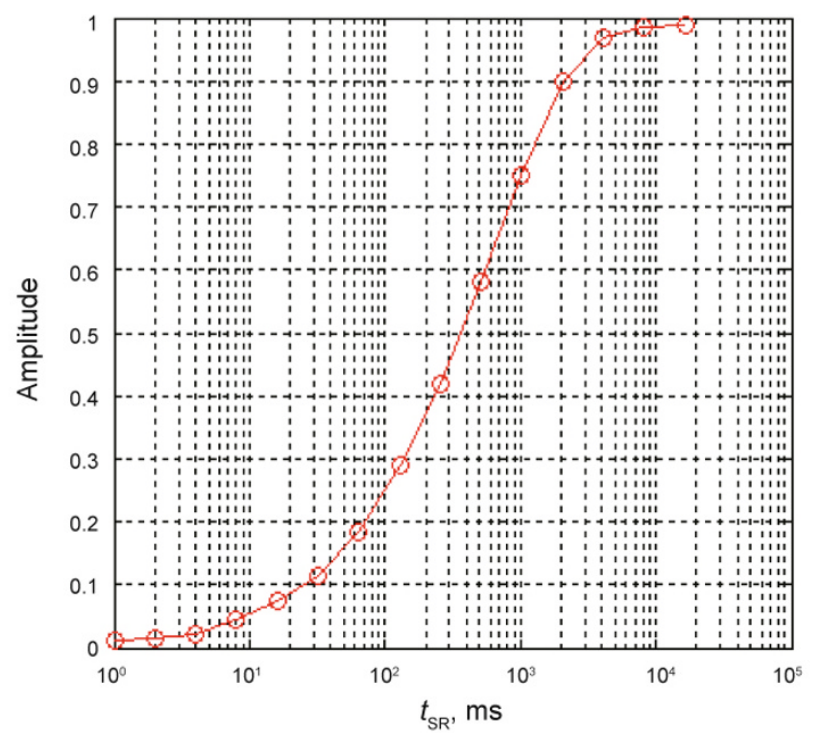

Fig. 12 Signal amplitude of the oil sample in the fluid analyzer changes with wait time $t_{\mathrm{SR}}$

According to the function of the resonance region, we divide it into two sections: transmitter section only and transmitter and receiver section. Suppose that fluid flowing into the sensor does not change in a total cycle time. We also require fluids that are not on resonance can not flow into transmitter and receiver section. We calculate the length of resonance region by the longest time to complete a sample (16,384 ms).

$$
\begin{aligned}
& \text { Coil 1: } L_{\mathrm{TS}}=v \times t=5 \mathrm{~cm} / \mathrm{s} \times 16.384 \mathrm{~s}=0.8192 \mathrm{~m} \\
& \text { Coil 2: } L_{\mathrm{TRS}}=\frac{1}{3} L_{\mathrm{S}}=0.27 \mathrm{~m}
\end{aligned}
$$

Considering design of the redundant length, the length of transmit coil is $0.82 \mathrm{~m}$, transmit and receive coil is $0.29 \mathrm{~m}$. Two coils are shown in Fig. 4. According to the length of two separate coils, the resonance region is $1.2 \mathrm{~m}$ long.

\section{Measurement results and discussion}

According to the designed parameters discussed above, we developed an NMR fluid analyzer sensor. Fig. 13(a) is a picture of the prototype sensor, in which three-part magnets are put into the high magnetically permeable tube with zero stray fields outside. We measured the $B_{0}$ field strength along the central axis from part $\mathrm{A}$ of the magnet to part $\mathrm{C}$. The 
measurement data was obtained every centimeter. (There is a high magnetically permeable steel ring at both ends when measured, reducing stray magnetic fields at the ends. So the field can be more uniform in the radial direction at the ends.) A gaussmeter (CH-1600, Beijing Ch-Hall Inc., Beijing) was used for measurements at room temperature. Fig. 13(b) is the curve based on the measured data. The maximum of $B_{0}$ field strength is 2,750 Gauss at $8 \mathrm{~cm}$, and the minimum is 970 Gauss at $21 \mathrm{~cm}$. While it is stable at about 1,270 Gauss at 33 $\mathrm{cm}$. Due to the inaccuracies of the magnetic materials and the magnet installation, the change of the $B_{0}$ field strength is about \pm 10 Gauss along the central axis in the measurement region. So the bandwidth of the $\mathrm{RF}$ coil should be $80 \mathrm{kHz}$ at least.

Fluids flow through the sensor in Fig. 13(a) at rate of $5 \mathrm{~cm} / \mathrm{s}$. Fluids with different $T_{1}$ will have different magnetization at $33 \mathrm{~cm}$ (as shown by red dashed curve 1 in
Fig. 13(c)). The relaxation time $T_{1}$ of fluids is among 2-3 s, so curve 1 shows that the magnetization of fluids at $33 \mathrm{~cm}$ is more than $95 \%$ of the final magnetization $\left(1.26 M_{0}\right)$.

Supposed that we replaced the designed pre-polarization magnet with the same length magnet (the field strength in the centre is 1,260 Gauss). The magnetization of fluids with the same rate at $33 \mathrm{~cm}$ is showed as green dashed curve 2, which shows that it is less than $95 \%$ of the final magnetization as the relaxation time $\mathrm{T} 1$ increases. In order to achieve $95 \%$ of the final magnetization, a longer magnet would be needed. That is to say, the NMR sensor was shortened for the pre-polarization magnet design.

Additionally, the actual magnetization of fluids at 33 cm (curve 1 in Fig. 13(c)) is less than the ideal simulation magnetization compared to Fig. 10 because of the inaccuracies, such as magnetic material, but the results still meet the design requirements.

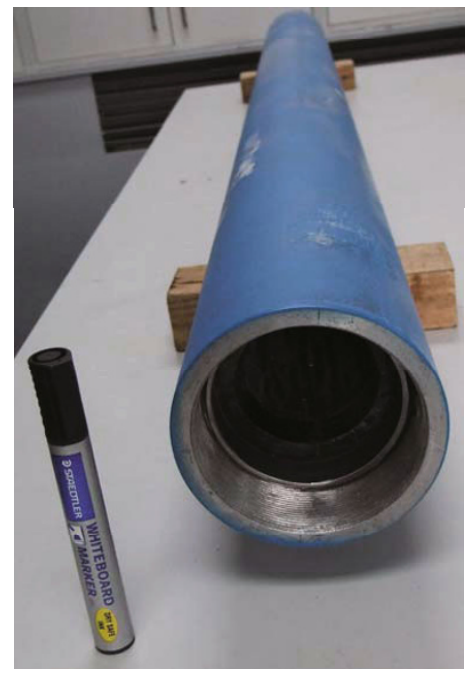

a

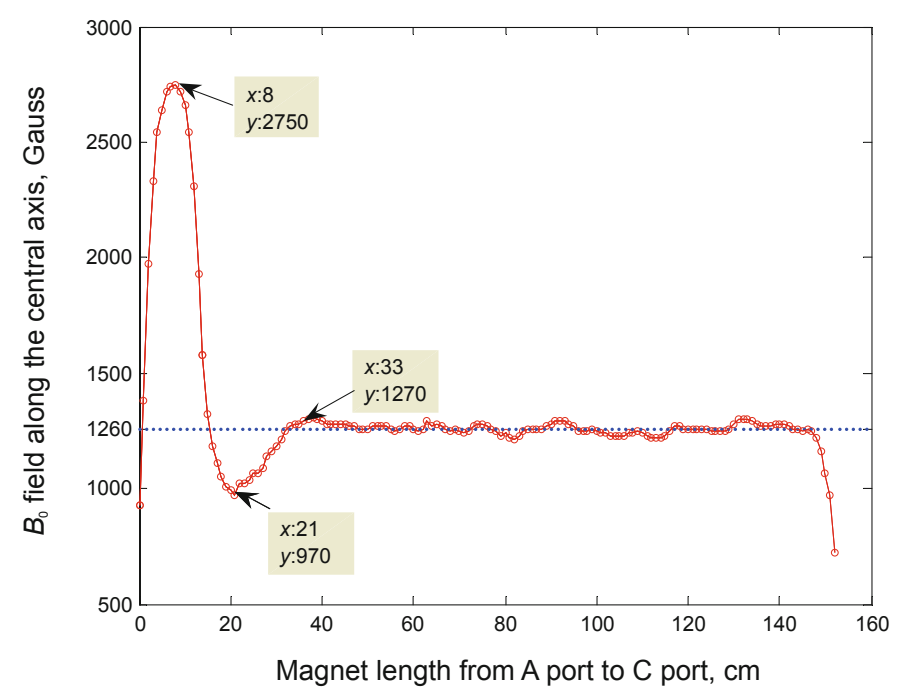

b

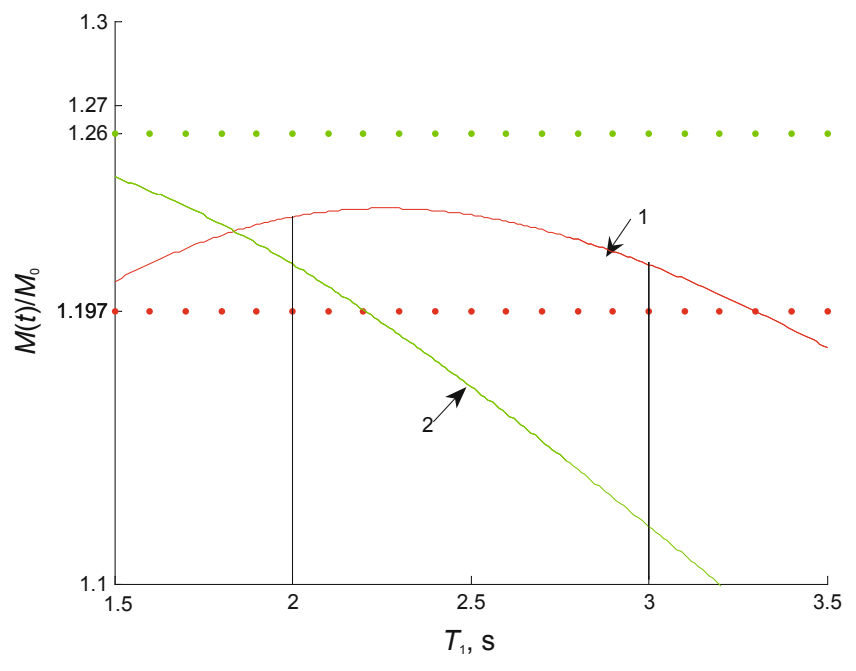

C
Fig. 13 The magnets and the measurement results. (a) A picture of the prototype sensor; (b) the measurement data shows that the magnetic field $B_{0}$ changes along the central axis; (c) the magnetization of fluids is compared in two cases. The green and red dot lines are 95\% of the final magnetization and the final magnetization respectively. The red dashed curve 1 shows that fluids with different $T_{1}$ obtain different magnetization when they pass the designed pre-polarization magnet. The red dashed curve 2 indicates a hypothetical situation

\section{Conclusions}

The NMR fluid analysis laboratory is a multi-functional tool, which can be used to analyze reservoir fluids in situ and in real-time. This paper describes the principle of the NMR fluid analysis laboratory when combined with RDT and its system structure. It has mainly researched its sensor. First, fluids were polarized when they passed the pre-polarization 
magnet. A RF field with a frequency $5.36 \mathrm{MHz}$ was applied to the fluids in the measurement region. The relaxation characteristics of fluids could be measured with a pulse sequence.

This paper reports the magnet structure and the design of magnet length. We utilized a low carbon steel tube with high magnetic permeability to form a large homogeneous region in the center. There is a zero stray field outside the tube. Finite element software was employed to do numerical simulation and optimize design parameters. According to the Bloch equation, a method was proposed to determine the optimal design of each part of the magnet length. This method is suitable for calculating the magnet length in LWD NMR or wireline NMR and it can also be used to design the sensor in on-line NMR detection.

The measurement results indicate that the magnet design is reasonable. An appropriate length of the pre-polarization magnet could improve the magnetization of fluids. The signal to noise ratio (SNR) was improved, as the SNR is proportional to the magnetization. In addition, the NMR sensor was shortened for the pre-polarization magnet design, and the weight of the logging tool was lowered. These could reduce difficulties of logging operations.

\section{Acknowledgements}

Financial support from the National Science Foundation of China (Grant No. 41074102) and the China International Science and Technology Cooperation (Grant No. 2009DFA61030) is acknowledged.

\section{References}

Akkurt R, Fransson C M, Witkowsky J M, et al. Fluid sampling and interpretation with the downhole NMR fluid analyzer. SPE Annual Technical Conference and Exhibition, 26-29 September 2004, Houston, Texas, U.S.A. (SPE 90971)

Blades T and Prammer M G. Nuclear magnetic resonance sensing apparatus and techniques for downhole measurements. U.S. Patent, US006111408A, 2000-08-29
Blümich B, Casanova F, Dabrowski M, et al. Small-scale instrumentation for nuclear magnetic resonance of porous media. New Journal of Physics. 2011. 13(1): 1-15

Buess M L and Petersen G L. Acoustic ringing effects in pulsed nuclear magnetic resonance probes. Review of Scientific Instruments. 1978. 49(8): 1151-1155

Coates G R, Xiao L Z and Prammer M G. NMR Logging Principles and Applications. America: Halliburton Energy Services Press. 1999. 2628

Dong C, O'Keefe M, Elshahawi H, et al. New downhole fluid analyzer tool for improved reservoir characterization. SPE Offshore Technology Conference, 4-7 September 2007, Aberdeen, Scotland, U.K. (SPE 108566)

Edwards C M, Fanini O N and Forgang S W. Nuclear magnetic resonance fluid characterization apparatus and method for using with electric wireline formation testing instruments. U.S. Patent, US006111409A, 2000-08-29

Fukushima E and Roeder S B W. Spurious ringing in pulse NMR. Journal of Magnetic Resonance. 1979. 33: 199-203

Joo T O. Measure instrument and method. U.S. Patent, US7501819B2, 2009-03-10

Luong B, Goswami J C, Sezginer A, et al. Optimal control technique for magnet design in inside-out nuclear magnetic resonance. IEEE Transactions on Magnetics. 2001. 37(2): 1015-1023

Miller M N, Prammer M G and Huber M. Apparatus and method for magnetic resonance measurements in an interior volume. U.S. Patent, US20020097051A1, 2002-07-25

Minh C C, Weinheber P, Wichers W, et al. Using the continuous NMR fluid properties scan to optimize sampling with wireline formation testers. SPE Annual Technical Conference and Exhibition, 21-24 September 2008, Denver, Colorado, U.S.A. (SPE 115822)

Prammer M G, Bouton J and Masak P C. The downhole NMR fluid analyzer. SPWLA 42nd Annual Symposium, 17-20 June 2001, Houston, Texas, U.S.A.

Prammer M G, Bouton J C and Masak P. Magnetic resonance fluid analysis apparatus and method. U.S. Patent, US20020140425A1, 2002-10-03

Seifert D J, Akkurt R, Al-Dossary S, et al. Nuclear magnetic resonance logging; while drilling, wireline and fluid sampling. SPE 15th Middle East Oil \& Gas Show and Conference, 11-14 March 2007, Kingdom of Bahrain (SPE 105605)

(Edited by Hao Jie) 